\title{
Nonalcoholic steatohepatitis
}

\section{A scientometric analysis of publications during 1980-2018}

\author{
Anca Trifan, MD, PhD, FRCP ${ }^{a, b}$, Carol Stanciu, MD, PhD, FRCP ${ }^{a, b}{ }^{*}$, Monica Jurcău, MD ${ }^{b}$, \\ Sebastian Zenovia, MD ${ }^{\mathrm{b}}$, Georgiana Frunzuc, $\mathrm{MD}^{\mathrm{b}}$, Daniel Timofte, MD, PhD ${ }^{\mathrm{a}, \mathrm{c}}$
}

\begin{abstract}
Background: Nonalcoholic steatohepatitis (NASH) is a subtype of non-alcoholic fatty liver disease (NAFLD) with a potentially progressive course to liver fibrosis, cirrhosis with its complications, or even hepatocellular carcinoma. NAFLD is a rapidly growing chronic liver disease, with a global prevalence of about 25\%, with a significant increase in the last 2 decades, changing the landscape of hepatology. This study aimed to undertake a bibliometric global analysis of research literature focusing on NASH.

Methods: We searched the Scopus database to identify all articles pertaining to "non-alcoholic steatohepatitis" or "NASH" - the 2 keywords used to search in the title or abstract within the time period 1980 to 2018. The collected data included document type, author, journal, publication year, citation reports, country, and were analyzed using Microsoft Excel and Microsoft Word.

Results: A total number of 6632 articles published in 1355 journals were retrieved. English was the predominant language of publication, USA being the most productive with 1937 articles published (29.2\% of the total number of publications), followed by Japan with 909 , representing $13.7 \%$ of publications. Hepatology, Journal of Hepatology and World Journal of Gastroenterology were the most active journals. Research articles were the most common type of publications (4524; $68.22 \%)$, followed by review articles (1359; 20.49\%). The total number of citations received by all publications was 274,041 , with an average of 41.32 per article (range: 0 4384). The average number of authors per article has increased in the last 2 decades, whereas the trend of single- (or few) authored publications has decreased.
\end{abstract}

Conclusion: This study indicates that NASH is a significant topic in the hepatology research, as proved by the huge number of publications, recording an exponential growth in the last 2 decades. The USA stands out as by far the most productive country.

Abbreviations: NAFLD = nonalcoholic fatty liver disease, $\mathrm{NASH}=$ nonalcoholic steatohepatitis.

Keywords: bibliometrics, nonalcoholic fatty liver disease, nonalcoholic steatohepatitis, scientometrics

\section{Introduction}

Nonalcoholic steatohepatitis (NASH), a subtype of nonalcoholic fatty liver disease (NAFLD), has become a major healthcare problem worldwide due to its high prevalence and potentially progressive course towards liver fibrosis, cirrhosis, hepatocellular carcinoma, and liver transplantation. Until 5 years ago, viral hepatitis (B and C) had been the focus of clinical research in hepatology. The advent of therapies that supress hepatitis B virus

\section{Editor: Giovanni Tarantino.}

The authors have no funding and conflicts of interest to disclose.

a "Grigore T. Popa" University of Medicine and Pharmacy, ${ }^{b}$ Institute of Gastroenterology and Hepatology, "Department of Surgery, "St. Spiridon" County Clinical Emergency Hospital, laşi, Romania.

* Correspondence: Carol Stanciu, Institute of Gastroenterology and Hepatology, "St. Spiridon" County Clinical Emergency Hospital, lasi 700111, Romania (e-mail: stanciucarol@yahoo.com).

Copyright () 2019 the Author(s). Published by Wolters Kluwer Health, Inc. This is an open access article distributed under the terms of the Creative Commons Attribution-Non Commercial License 4.0 (CCBY-NC), where it is permissible to download, share, remix, transform, and buildup the work provided it is properly cited. The work cannot be used commercially without permission from the journal.

How to cite this article: Trifan A, Stanciu C, Jurcău M, Zenovia S, Frunzuc G, Timofte D. Nonalcoholic steatohepatitis: A scientometric analysis of publications during 1980-2018. Medicine 2019;98:50(e18221).

Received: 11 July 2019 / Received in final form: 16 October 2019 / Accepted: 4 November 2019

http://dx.doi.org/10.1097/MD.0000000000018221 replication and the highly effective direct-acting antivirals, curing hepatitis $\mathrm{C}$ virus-infection in almost all patients and is looking forward to eliminating this infection in $2030,{ }^{[1]}$ the focus has been switched towards NAFLD, and to one of its 2 types, NASH in particular, which has replaced viral hepatitis, and thus, changing the perspective in hepatology.

NAFLD is the chronic liver disease with the fastest growth, reaching a global prevalence of about $25 \%$, while the prevalence of NASH among NAFLD patients ranges from $10 \%$ to $59 \%$. In the general population, the prevalence of NASH is between $1.5 \%$ and $6.5 \%$, and has been increasing in the last decade worldwide in parallel with the prevalence of obesity and diabetes. ${ }^{[2,3]}$

Scientometrics or bibliometrics are methodological approaches aimed to provide quantitative and qualitative analysis of scientific literature on a large variety of topics (medical or nonmedical). ${ }^{[4]}$ To our knowledge, no bibliometric studies have been carried out so far at the global level to assess NASH exclusively.

The aim of this study was to provide accurate data on worldwide research productivity and publication trends in the field of NASH, indexed in the Scopus database, using the bibliometric approach.

\section{Methods}

The data of this study were retrieved from the Scopus database for the period 1980 to 2018. The Scopus database was selected for this bibliometric study as it provides detailed information about ranking of authors, country affiliation, journals, and 
citations. Most of the bibliometric indicators were presented in table or figure format. We searched the Scopus database to identify all articles pertaining to "non-alcoholic steatohepatitis" or "NASH" - the 2 keywords used to search in title or abstract. We included studies published in English, German and French languages. The collected data by 3 authors (MJ, SZ, GF) included number of articles published annually, document type, Hirsch index ( $h$-index), authorship, source journal, publication year, number of citations, country, and were analyzed using Microsoft Excel and Microsoft Word. We included original articles, reviews, meta-analysis, editorials, and letters. We calculated the number of citations for all included articles and obtained the journals' impact factors (from SCImago Journal \& Country Rank). As the number of citations could differ from 1 day to another, citation analyses were made on the same day (May 25, 2019).

All data were recovered from the Scopus database and it did not require any approval from an ethics committee.

\section{Results}

A total of 6632 NASH-related publications identified in the Scopus database were published in 1355 journals between 1980 and 2018. The first article related to NASH was published by Ludwig et al in Mayo Clinic Proceedings, $1980 .{ }^{[5]}$ Since then, the number of publications on NASH has slowly increased on a yearly basis, until 2000, followed by an exponential growth in the past 2 decades (Fig. 1). The number of published studies during the last 3 years (2016-2018) accounted for 2025 (30.53\%) of the total publications.

The original article was the most common publication type, comprising $4524(68.22 \%)$ of the total publications, followed by review articles $(1359 ; 20.49 \%)$, while English $(5921 ; 89.27 \%)$ was the primary language of articles, followed by German (100; $1.5 \%)$ and French $(62 ; 0.93 \%)$.

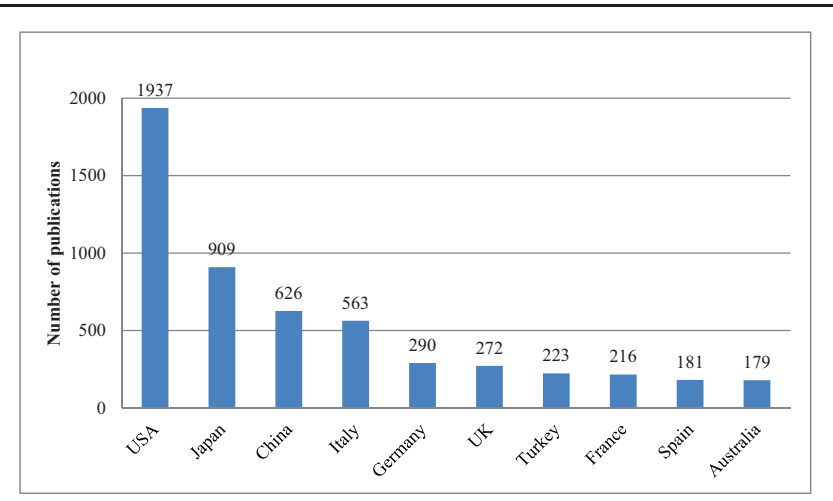

Figure 2. Top 10 most productive countries.

Authors from 88 different countries contributed to publications, with the USA occupying the first place $(1937 ; 29.2 \%)$, followed by Japan $(909 ; 13.7 \%)$, and China $(626 ; 9.43 \%)$. The USA was not only the country with highest number of publications, but also with the highest $h$-index of 166 . Top 10 most productive countries are shown in Figure 2.

Table 1 shows the top 10 active journals in publishing articles regarding NASH, with Hepatology as the most productive journal (333; 5.02\%), followed by Journal of Hepatology (191; $2.87 \%$ ), World Journal of Gastroenterology (190; $2.86 \%$ ), Hepatology Research $(143 ; 2.15 \%)$, Journal of Gastroenterology and Hepatology Australia (131; 1.97\%), PloS One (130; 1.96\%), Liver International $(121 ; 1.82 \%)$, Digestive Diseases and Sciences (108; 1.62\%), Gastroenterology $(90 ; 1.35 \%)$, Alimentary Pharmacology and Therapeutics $(79 ; 1.19 \%)$. All the top 10 journals have $h$-index $\geq 66$ (SCImago Journal \& Country Rank).

The total number of citations for publications was 274,041 with an average of 41.32 per publication (ranging from 0 to

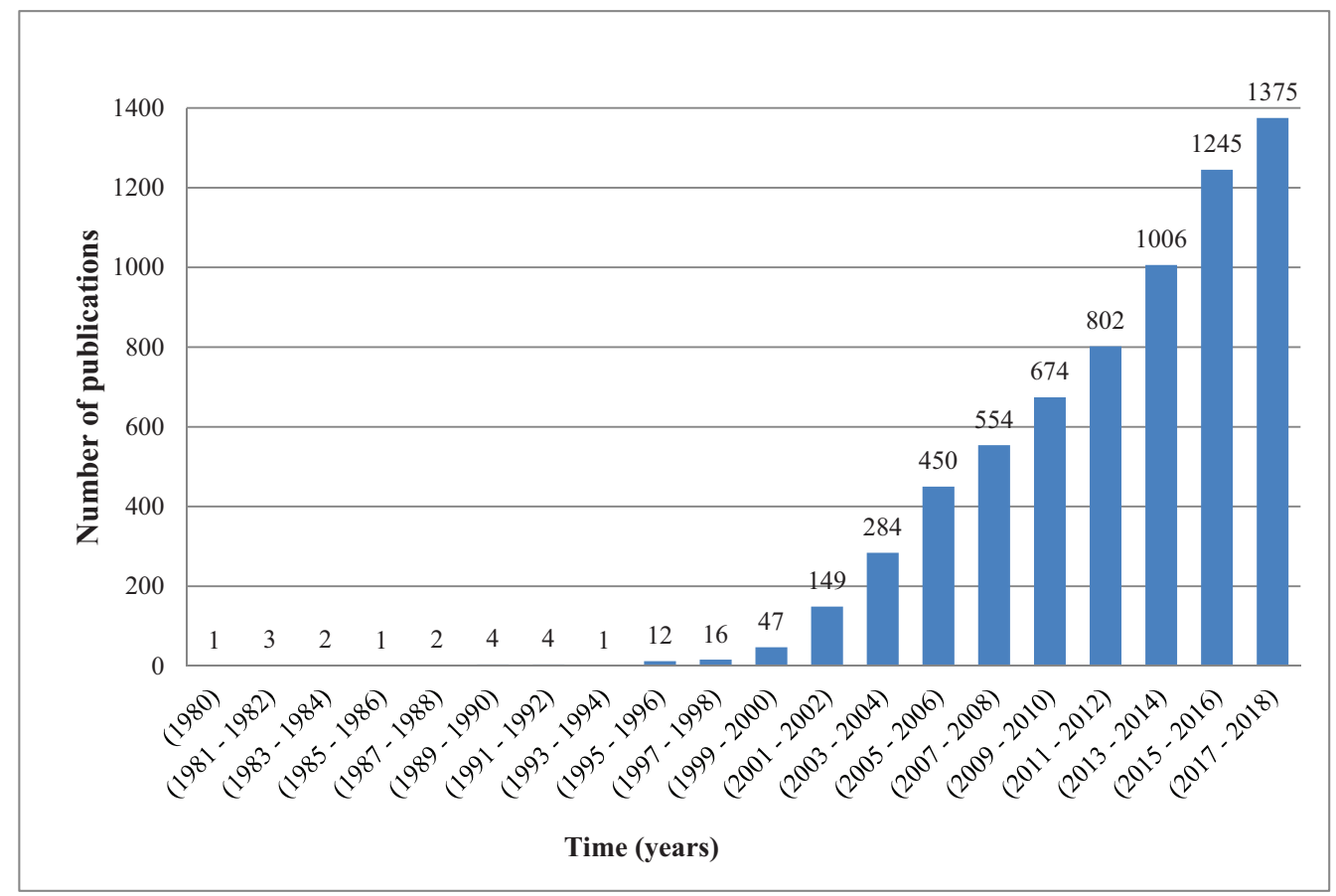

Figure 1. Publications grouped in $2 \mathrm{yr}$ intervals. 


\section{Table 1}

Top 10 most productive journals.

\begin{tabular}{|c|c|c|c|c|}
\hline Rank & Journal & N (\%) & Impact factor & $H$-index \\
\hline 1 & Hepatology & $333(5.02 \%)$ & 14.079 & 317 \\
\hline 2 & Journal of Hepatology & $191(2.87 \%)$ & 14.911 & 216 \\
\hline 3 & World Journal of Gastroenterology & $190(2.86 \%)$ & 3.300 & 129 \\
\hline 5 & Journal of Gastroenterology and Hepatology (Australia) & $131(1.97 \%)$ & 3.483 & 117 \\
\hline 6 & PLoS One & $130(1.96 \%)$ & 2.766 & 268 \\
\hline 7 & Liver International & $121(1.82 \%)$ & 4.500 & 98 \\
\hline 10 & Alimentary Pharmacology and Therapeutics & $79(1.19 \%)$ & 7.357 & 159 \\
\hline
\end{tabular}

4384). The $h$-index of the 6632 articles published from 1980 to 2018 was 217 . A number of 615 publications were each cited at least 100 times. The ranking of the top 20 cited publications worldwide associated with the use of NASH are shown in Table 2 (13 originated from USA, 3 from Australia and 1 from Italy, Sweden, Turkey, and France, each). ${ }^{[5-24]}$
The authors from top 20 articles were from 6 countries (USA, Italy, Australia, Sweden, Turkey, and France) published in 6 journals: Gastroenterology (8), Hepatology (7), New England Journal of Medicine (2), American Journal of Gastroenterology (1), Alimentary Pharmacology and Therapeutics (1), Nature (1).

\section{Table 2}

Top 20 publications selected by citations.

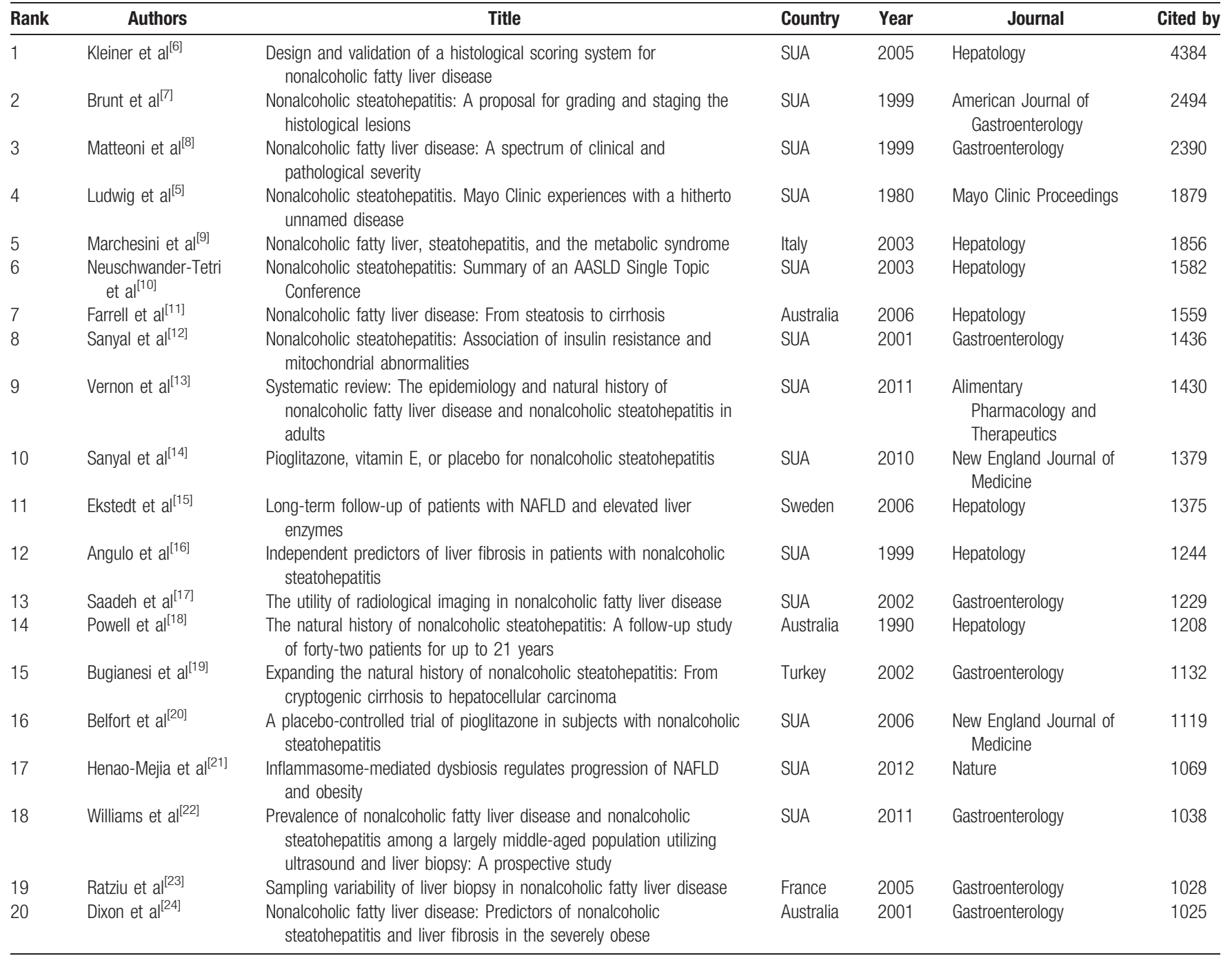

NAFLD $=$ nonalcoholic fatty liver disease, AASLD = American Association for the Study of Liver Diseases 
The number of authors who participated in published documents was 3272. The mean number of authors per article increased with time (4.38 in the year 2000, 5.26 in 2005, 6.32 in $2010,6.99$ in 2015, 7.66 in 2018), whereas our study showed a decreasing trend of single-authored publications. At the same time, the number (percentage) of multi-authored publications increased from $24(82.76 \%)$ in the year 2000, up to 678 $(94.29 \%)$ in 2018.

\section{Discussion}

For this bibliometric study, we selected the Scopus database as the source to retrieve all data regarding the research output in the field of NASH. The Scopus database is the largest and one of the most reliable databases for publications and citations, combining in characteristics both PubMed and Web of Science ${ }^{[25,26]}$ and providing quantitative (number of studies) as well as qualitative data - impact ( $h$-index).

We included a total of 6632 publications regarding NASH in English, French, and German. The number of writings has been growing continuously, with an exponential increase in the last 2 decades, demonstrating that NASH has been and remains a hot issue in scientific research in the field of hepatology. The USA, Japan, and China were found to be the most productive countries in this aspect.

The prevalence of NASH has increased worldwide over the last 2 decades, paralleling a soaring prevalence in obesity, diabetes mellitus, and metabolic syndrome, all 3 being the most important risk factors for $\mathrm{NASH}^{[27]}$ and we should expected to witness a higher prevalence of this disease in the future as some etiological factors of NASH such as genetics, high-fat diet, and sedentary lifestyle will continue to dominate our life.

Our study has some strengths and several limitations. Thus, we used the Scopus database as the source to retrieve all publications pertaining NASH, as it is the most recognized database for reviewing scientific literature on a certain field of research. This study is the first to analyze research activity in the field of NASH, showing an exponential increase in the number of publications. However, referring to limitations, we should mention that by using the Scopus database alone, the contribution of other databases may have been underestimated. Also, some publications did not contain NASH in the publication titles, but they mentioned it inside the text (eg, NAFLD); such publications were not included in this study. In addition, some peer-review journals from Asian and African countries are not indexed in Scopus.

To our knowledge, this is the first bibliometric study to analyze and quantify global research productivity related to NASH. However, it should be mentioned that Zhang et al recently published a bibliometric analysis and systematic review of global publications regarding NAFLD with its 2 components - NAFL and $\mathrm{NASH},{ }^{[28]}$ whereas our study included only publications pertaining to NASH. This is the difference between the 2 studies and we cannot compare the data regarding only NASH with those concerning NAFLD, since the use of 2 terms (NASH and NAFLD) may lead to statistic bias.

\section{Conclusion}

This study showed NASH to be a key topic in the hepatology research output and offers researchers and clinicians a leading tour in this area of research.

\section{Author contributions}

Conceptualization: Anca Trifan.

Data curation: Monica Jurcău, Sebastian Zenovia, Georgiana Frunzuc.

Formal analysis: Anca Trifan.

Investigation: Daniel Timofte.

Methodology: Daniel Timofte.

Project administration: Carol Stanciu.

Software: Monica Jurcău.

Supervision: Carol Stanciu.

Validation: Carol Stanciu.

Writing - original draft: Monica Jurcău, Sebastian Zenovia, Georgiana Frunzuc.

Writing - review and editing: Anca Trifan.

\section{References}

[1] World Health Organization. Global Health Sector Strategy on Viral hepatitis, 2016-2021. Geneva: 2016. Available at: http://apps.who.int/ iris/bitstream/10665/246177/1/WHO-HIV2016.06-eng.pdf?ua=1

[2] Younossi ZM, Koening AB, Abdelatif D, et al. Global epidemiology of nonalcoholic fatty liver disease - meta-analytic assessment of prevalence, incidence, and outcomes. Hepatology 2016;64:73-84.

[3] Younossi ZM, Blissett D, Blisset R, et al. The economic and clinical burden of nonalcoholic fatty liver disease in the United States and Europe. Hepatology 2016;64:1577-86.

[4] Lutman M. Bibliometric analysis as a measure of scientific output. Br J Audiol 1992;26:323-4.

[5] Ludwig J, Viggiano TR, McGill DB, et al. Nonalcoholic steatohepatitis. Mayo Clinic experiences with a hitherto unnamed disease. Mayo Clinic Proc 1980;55:434-8.

[6] Kleiner DE, Brunt EM, Van Natta M, et al. Design and validation of a histological scoring system for nonalcoholic fatty liver disease. Hepatology 2005;41:1313-21.

[7] Brunt EM, Janney CG, Di Bisceglie AM. Nonalcoholic steatohepatitis: a proposal for grading and staging the histological lesions. Am J Gastroenterol 1999;94:2467-74.

[8] Matteoni CA, Younossi ZM, Gramlich T. Nonalcoholic fatty liver disease: a spectrum of clinical and pathological severity. Gastroenterology 1999;116:1413-9.

[9] Marchesini G, Bugianesi E, Forlani G, et al. Nonalcoholic fatty liver, steatohepatitis, and the metabolic syndrome. Hepatology 2003;37: 917-23.

[10] Neuschwander-Tetri BA, Caldwell SH. Nonalcoholic steatohepatitis: summary of an AASLD single topic conference. Hepatology 2003;37:1202-19.

[11] Farrell GC, Larter CZ. Nonalcoholic fatty liver disease: from steatosis to cirrhosis. Hepatology 2006;43:99-112.

[12] Sanyal AJ, Campbell-Sargent C, Mirshahi F, et al. Nonalcoholic steatohepatitis: association of insulin resistance and mitochondrial abnormalities. Gastroenterology 2001;120:183-192.

[13] Vernon G, Baranova A, Younossi ZM. Systematic review: the epidemiology and natural history of non-alcoholic fatty liver disease and non-alcoholic steatohepatitis in adults. Aliment Pharmacol Therap 2011;34:274-85.

[14] Sanyal AJ, Chalasani N, Kowdley KV, et al. Pioglitazone, vitamin E, or placebo for nonalcoholic steatohepatitis. N Engl J Med 2010;362:1675-85.

[15] Ekstedt M, Franzén LE, Mathiesen UL, et al. Long-term follow-up of patients with NAFLD and elevated liver enzymes. Hepatology 2006;44:865-73.

[16] Angulo P, Keach JC, Batts KP, et al. Independent predictors of liver fibrosis in patients with nonalcoholic steatohepatitis. Hepatology 1999;30:1356-62.

[17] Saadeh S, Younossi ZM, Remer EM, et al. The utility of radiological imaging in nonalcoholic fatty liver disease. Gastroenterology 2002;123:745-50.

[18] Powell EE, Cooksley WGE, Hanson R, et al. The natural history of nonalcoholic steatohepatitis: a follow-up study of forty-two patients for up to 21 years. Hepatology 1990;11:74-80. 
[19] Bugianesi E, Leone N, Vanni E, et al. Expanding the natural history of nonalcoholic steatohepatitis: from cryptogenic cirrhosis to hepatocellular carcinoma. Gastroenterology 2002;123:134-40.

[20] Belfort R, Harrison SA, Brown K, et al. A placebo-controlled trial of pioglitazone in subjects with nonalcoholic steatohepatitis. N Engl J Med 2006;355:2297-307.

[21] Henao-Mejia J, Elinav E, Jin C, et al. Inflammasome-mediated dysbiosis regulates progression of NAFLD and obesity. Nature 2012;482:179-85.

[22] Williams CD, Stengel J, Asike MI, et al. Prevalence of nonalcoholic fatty liver disease and nonalcoholic steatohepatitis among a largely middleaged population utilizing ultrasound and liver biopsy: a prospective study. Gastroenterology 2011;140:124-31.

[23] Ratziu V, Charlotte F, Heurtier A, et al. Sampling variability of liver biopsy in nonalcoholic fatty liver disease. Gastroenterology 2005;128:1898-906.
[24] Dixon JB, Bhathal PS, O'Brien PE. Nonalcoholic fatty liver disease: predictors of nonalcoholic steatohepatitis and liver fibrosis in the severely obese. Gastroenterology 2001;121:91-100.

[25] Zyoud SH, Waring WS, Sweileh WM, et al. Global research trends in lithium toxicity from 1913 to 2015: a bibliometric analysis. Basic Clin Pharmacol Toxicol 2017;121:67-73.

[26] Palomo J, Figueroa-Domecq C, Laguna P. Women, peace and security state-of-art: a bibliometric analysis in social sciences based on SCOPUS database. Scientometrics 2017;113:123-48.

[27] Younossi Z, Tacke F, Arrese M, et al. Global perspectives on nonalcoholic fatty liver disease and nonalcoholic steatohepatitis. Hepatology 2019;69:2672-82.

[28] Zhang TS, Qin HL, Wang T, et al. Global publication trends and research hotspots of nonalcoholic fatty liver disease: a bibliometric analysis and systematic review. SpringerPlus 2015;4:776. 